\title{
A holistic approach to fieldwork through balanced reflective practice
}

Erik Blair ${ }^{\mathrm{a}}$ and Amy Deacon ${ }^{\mathrm{b}}$

${ }^{a}$ Centre for Excellence in Teaching and Learning, The University of the West Indies, St Augustine, Trinidad and Tobago; ${ }^{b}$ Centre for Biological Diversity, School of Biology, University of St Andrews, Scotland

\footnotetext{
Reflective practice is well-established as a tool for practitioner development in areas such as nursing, social work and education. Reflection involves the integration of theoretical constructs and practical action; therefore it seems somewhat ironic that there is little written on reflective practice within the natural sciences - where theory and action are often juxtaposed. This paper attempts to address this gap through examining biological fieldwork in relation to a balanced system of reflection that embraces the cognitive, psychomotor, affective and conative aspects of practice. A model of reflective practice that asks practitioners to log their reflections against these four domains was applied to a biodiversity survey of tropical mountain streams in Trinidad. It was found that there is clear evidence that biological fieldwork can embrace a reflective methodology, and that reflective practice can be used in fieldwork as a tool for making explicit that which is already implicit. A holistic vision of fieldwork is sketched out here, where the introduction of a balanced model of reflective practice can support an approach that moves beyond the consideration of the environment and the researcher as two separate entities and, instead, considers the relationship between environment and researcher.
}

Keywords: Vitruvian Reflection; biology; reflective framework, holistic, domains of learning

\section{Introduction}

Reflective practice is an established practice in disciplines such as teacher training, childhood education, nursing, physical therapy, and psychology (Dyment \& O'Connell, 2010); however in other areas it is relatively unknown. A review of the abstracts of 205 articles from this journal (from issue 12(1) to issue 15(2)), found that they covered, inter alia, health care, education, social care, psychology, coaching, supervision and managerial development. Here we see rich and varied discussion in disciplines under the broad umbrella of the social sciences, but a paucity of discussion on reflection in the natural sciences. We found articles that discussed reflection in relation to the teaching of the natural sciences, but in each of these cases the emphasis was on reflection in relation to pedagogical matters rather than reflection on scientific processes per se. A literature search of other journals (from the natural and social sciences) 
showed similar results: reflection is rife in some disciplines and absent in others. We were able to identify two anomalies: Hazzan and Tomayko (2004) examined reflective practice in relation to software design, and Fazey, Fazey and Fazey (2005) discussed how an individual's reflections on their experiences can help them develop a better understanding of biological systems. Hazzan and Tomayko's application of Schön's work and Fazey, Fazey and Fazey's examination of the experimental/experiential nexus, gave us scope to think that there was room for the application of reflective practice in areas where it does not seem to have been applied before. Since one of the authors is an active biologist, we felt that there was room to apply a reflective practice framework to the area of biological fieldwork, so as to investigate the usability of reflective practice in this activity. There does seem to be some irony in the finding that reflective practice exists in medicine (e.g. Mann, Gordon \& MacLeod, 2009) but appears to be absent in the more experimental arm of natural science. Both medicine and biology have their roots in scientific reasoning, yet these cousin disciplines seem to have taken different approaches to the role of the individual within the reasoning process. This rift is investigated here through reconceptualising the actively enquiring individual in the biological fieldwork environment, and examining the value of reflection in such an environment.

For professional scientists, the utility of fieldwork is not up for debate; it is the means by which data is collected so that theories may be tested and discoveries made. It reinforces the vital connection between the intellectual pursuits of scientists and the real world (Noss, 1996). There are two main types of biological fieldwork: 1) as a means of collecting data; 2) as a teaching tool. A large literature exists on the latter, and fieldwork is widely acknowledged to be a useful pedagogical tool (see Scott, Goudler, Wheeler, Scoot, Tobin \& Marsham, 2011). However, given that fieldwork is fundamental to progress in many areas of research in biology, it is surprising that very little has been said about the link between the intellectual and the practical aspects of fieldwork and that the experimental/experiential nexus has not been considered in the same way it has in other disciplines. Anthropologists and ethnographers appear to have embraced reflective practice in their fieldwork, while biologists seem to have overlooked it. 
The idea of keeping a diary to reflect on fieldwork developments and data interpretation is certainly not new to biology; indeed, some of the most influential field biologists of all time used journals as a tool to reflect on their data and theories (e.g. Darwin, 1839; Wallace, 1889). However, as the discipline has moved toward a more structured scientific method over the last century, this more qualitative element appears to have been abandoned.

Fieldwork is established as an invaluable scientific tool; however, in problematising this area, we were forced to consider whether researchers in the field may be missing a developmental trick if they are not exposed to the possibilities of reflective practice. We postulate that it may be time for the reintroduction of a more reflective element to fieldwork in the biological sciences, not as a replacement for the quantitative approach that defines the discipline and is responsible for many significant developments in our knowledge, but rather to consider whether reflective practice may have something constructive to add to the process of conducting fieldwork and data collection.

In addressing this gap in the current construction of biological fieldwork, we decided to introduce a model of reflection into an ongoing study of biodiversity in the mountain streams of Trinidad. There were three key considerations that needed to be addressed: firstly, there should be no negative impact upon the project; secondly, the model of reflection had to be portable, and thirdly, since biological fieldwork relies on knowledge acquisition and application, the model of reflection needed to cover a range of domains of learning. Here we report on our examination of the experimental/experiential nexus of biological fieldwork and propose that the introduction of a model of balanced reflective practice could lead to a more holistic approach to fieldwork.

\section{Enacting and enhancing fieldwork}

Fieldwork 'addresses the complex variables involved in the interrelationships between living things and their environment' (Lock, 1998, p.633). Once field skills have been developed through training and observation, they are refined over time through practical application. Experts in the field receive and respond to feedback from their environment, and this iterative/experiential process makes them more 
adaptive to the needs of the ecological system (Fazey, Proust, Newell, Johnson \& Fazey, 2006).

Professional biological fieldwork is a complicated arena, and if properly formulated and enacted it can make for a deep learning experience (Kent, Golbertson \& Hunt, 1997; Higgit, 1996) that embraces many domains of learning (Scott et al, 2011).

Researchers in the field are not simply data-collecting automatons - they are active intellectuals involved in a meaning-making process and their considered judgements can enrich their professional practice. This experiential process is likely to enhance the quality of the fieldwork (Fazey, Fazey \& Fazey, 2005) and give impetus to professional development (Hays \& Gay, 2011). Professional development, through reflective practice, can also help connect theory and practice through an approach that is both affective and intellectual (Eisner \& Powell, 2002). However, since reflection is a complicated process involving 'numerous cognitive processes such as thinking, communicating, revising, and refining of observations and events' (Thorsen \& DeVore, 2013, p.96), this development may be restricted if effective reflective methods are not employed. Loughram (2002, p.42) reports that '[e]ffective reflective practice is drawn from the ability to frame and reframe the practice setting' and, as such, the potency of reflection is reduced if individuals are not able to critically problematise their situation through 'conscious reflection on the situation and, at the same time, on one's way of thinking and acting on it' (Schön, 1992, p.126). Here we see that reflective practice can be the motor that drives improvement but, for this to happen effectively, there needs to be a contextualised framework (Drummer, Cook, Parker, Barrett \& Hull, 2008).

Whilst there is a dearth of literature on reflective practice in relation to professional biological fieldwork, there is literature on reflection in relation to the teaching and/or learning of fieldwork (in biology and other related disciplines). Here we find that reflective journals have been used as a method of framing conscious reflection; reflective fieldwork diaries can encourage deep learning (McGuinness \& Simm, 2005), and can enhance meta-cognition (Mair, 2012). Reflective practice in such instances has been shown to enable the orchestration and employment of information and understanding (Levine, Kern \& Wright, 2008). The use of logged reflective accounts is not without question, and Clinton (1997) 
highlights the importance of understanding reflective accounts as a hermeneutic representation of activity and cognition rather than the full/true account. Smith (2011) goes further and reports that some would consider that critical personal reflection is not truly academic. However, such attacks on the reliability of reflective practice miss the central tenet of reflection: that reflective practice is a means of deliberately examining one's own practice in order to enhance personal expertise. Thompson and Pascal (2012, p.314) cite Schön (1983) in positing that reflective practice 'offers a practice more firmly rooted in the realities of practice, in which the "high ground" of the professional knowledge base offers helpful insights, but not simple direct lines of action for dealing with the "swampy lowlands of practice"'. In this regard, reflective practice is not validated through external conceits but is legitimised through the constancy of reflexivity.

The development of a researcher's understanding of environmental systems is a multi-faceted, experiential process and, in order to be effective, 'practice must be purposeful and fit in an overarching framework that includes planning, monitoring and reviewing' (Fazey, Fazey \& Fazey, 2005, online). Instead of considering the environment and the researcher's mind as two separate entities, there is room to consider how the environment is experienced by the researcher (Fazey \& Marton, 2002). The examination of this fieldwork/researcher relationship allows for the subjectification of fieldwork and the recognition of the role of the expert within the field. In examining the role of reflection at this nexus, we felt that the structured reflective approach could be applied to the work of the professional biological researcher. In order to do this, we needed a framework that was flexible and embraced the contextuality of practice in a number of environments.

As researchers, we come from different disciplines - education (Erik) and biological science (Amy) - therefore we held an initial research meeting so that we could develop a shared understanding of reflective practice before the data collection began (Thorpe, 2004). During and after this meeting we shared several papers on reflection and examined them, so that we were both grounded in the field and had a shared vision. In further discussions we examined how the perceived gap in fieldwork practice could be investigated and, in order to investigate the worth of reflective practice in biological fieldwork, we decided to introduce a model of reflective practice that was designed to be portable and applicable in a 
number of situations and embraced a range of domains, so that reflections on the researcher's environment were just as important as reflections that related to the researcher's cognition. Here we used the Vitruvian Reflection framework (Blair, 2011). Vitruvian Reflection (VR) is a framework for reflection that asks individuals to reflect on four specified aspects of their practice and, whilst it is suggested that this information should take the form of a 'conversation with oneself' (Blair, 2011, p.258), we felt that we needed to log information gathered through this framework so that we could analyse its worth as a tool for reflective practice in biological fieldwork. Other reflective frameworks were considered but did not quite meet the specific needs of this study. For instance, Griffin (2003) discusses the use of Critical Incidents as reflective tools that can give meaning to events. However, using a Critical Incident framework would mean recording reflections in 'rich and concrete detail' (p.210) and we felt that this would not be possible in the midst of biological fieldwork - where quick and focussed notes might be more appropriate. Other frameworks for analysing practice can involve detailed study after-the-fact. Deaton (2012) asked science teachers to analyse video footage of their teaching and record their reflections under a number of headings. Since our research took place in a situation where video recording would be very difficult, we felt that this approach would not work. McCollum (2002) illustrates how a reflective framework for teaching physical education might be used effectively. This approach offered us some flexibility as it was suitable to reflection in-situ; however, its pedagogical nature meant that it was more suited to teacher reflection. In the end, the VR model offered a framework that was suitable for the field as it could be completed quickly and without additional direction or equipment.

The VR model was developed as a proportionate and balanced model of reflection, and proposed as a means of addressing reflection within a number of non-traditional education settings. The model was developed for practitioners in the Post-Compulsory Education and Training sector where lecturers teach in disciplines as diverse as motor vehicle studies, hairdressing, child care, catering, accountancy and adult literacy. The VR model asks individuals to reflect on their practice in relation to the four domains of learning developed in the writings of Benjamin Bloom and his colleagues (Bloom, Englehart, Furst, Hill \& Krathwohl. 1956; Krathwohl, Bloom \& Masia, 1964). These four domains relate to the cognitive, 
psychomotor, affective and conative dimensions of learning, and place reflection in a space where the consideration of what it is to 'understand' something is more than the collection of data/knowledge. Reflection on the cognitive domain involves the practitioner questioning what they know; how this relates to their previous knowledge, and developing new schema. The VR model was developed for use in a range of environments and the psychomotor aspect considers reflection on the movements, actions and interaction of the practitioner to be significant to the improvement of future practice. Reflection on the affective domain helps tie the activity being undertaken with how the practitioner feels about it - here learning is subjective and has specific emotive qualities. The final aspect of the VR model involves reflection on the effort that is required to make sense of a situation, and proposes that practitioners should be aware of the impact of the levels of their activity/passivity. By recognising that educational experiences involve knowledge, effort, emotion and persistence, the four domains that make up the VR model work together to create a more holistic understanding of what it is to be a practitioner within a specific context. Therefore, based on our review of reflective frameworks, in conjunction with the particular requirements of fieldwork, we felt that the VR model would be a balanced and practical tool for examining reflective practice in biological fieldwork.

\section{Methodology}

\section{Framing the study}

This study uses Amy's reflective accounts as qualitative data. It does not claim to develop grand theory or a universal answer. Instead the findings and analyses are specific to this one case. It is recognised that such studies are not easily generalisable; however, there are often common threads of experience that can be highlighted through such deliberations, and aspects of this study will, no doubt, speak to many involved in similar types of context-specific practice. The application of the reflective practice to biological fieldwork is certainly a repeatable experiment and the use of the VR model as a reflective tool is, likewise, transferable to other areas of practice. In framing this study thus, we feel that we can offer a valid and reliable account of our attempt to develop a holistic approach to fieldwork through balanced 
reflective practice. In utilising the VR model we developed a data collection tool that asked the following four questions in relation to biological fieldwork:

1) Cognitive: What are your 'expert' thoughts about this site?

2) Psychomotor: How do you proceed physically within this site?

3) Affective: How do you feel about this site?

4) Conative: How much effort will you give to this site?

\section{The research context}

In this instance, fieldwork is biodiversity surveys in tropical mountain streams of Trinidad in the Caribbean. The aim of the fieldwork is to address theoretical ecological questions. In her capacity as a biological research scientist, Amy is responsible for planning and conducting regular fieldwork surveys with a team of three assistants. This particular study is a longitudinal ( 5 year) project, with multiple sessions of fieldwork spread over this period. The fieldwork involves transporting equipment from a vehicle to the riverbank, before blocking a section of stream with large nets. Seine nets and electrofishing equipment are then used to catch all of the fish within that section. Fish are identified and weighed before being returned to the river. Samples from the stream bed are collected for later analysis in the laboratory. Physical measurements including water temperature and $\mathrm{pH}$ are also taken. Most of these tasks involve walking in and alongside the rivers, in various weather conditions, as well as communicating and cooperating with colleagues.

Every three months, the same sites are surveyed using the same methods, in order to monitor changes in biological diversity over time. These sites consist of a series of pairs, each pair including a recreationally-used site and a more pristine site. By comparing the more-disturbed and less-disturbed sites the impact of human disturbance on natural communities over time can be assessed. This fieldwork project can be considered fairly typical of biological fieldwork in general, in that it requires constant adjustment of actions and methods depending on the particular physical and social conditions. As such, 
this project provides an excellent opportunity to evaluate the application of reflective practice during a series of authentic biological fieldwork trips.

\section{Data collection}

Here, data is drawn from one set of eight trips, which took place during a three week period in April-May 2014. On each trip, two sites were visited (one pair). As each site has different biological and physical conditions and associated challenges, spanning the data collection over an entire set of eight trips increased the range of circumstances to which the approach could be applied, and subsequently evaluated under. A separate set of reflective notes was made for each site to allow comparisons between sites. A single data collection sheet was developed that allowed for reflective comments to be made against the four aspects of the VR model: conative, affective, cognitive and psychomotor (see Appendix A). A new sheet was used for each of the eight trips for convenience, and so that Amy's reflections would not be influenced by previous days' notes. We were concerned that the task should not simply be a matter of jotting down notes in a pre-determined order but that it should involve a more active approach. We also felt that it was important that no one domain should be seen to be more important than another. Therefore, the order in which the four VR aspects appeared on the sheet each day varied systematically, with the intention of minimising any bias caused by the order in which they were considered and to discourage an autopilot response (see Appendix B). The majority of the reflection took place in action - immediately before, during and immediately after the surveys; this involved completing each section of the data sheet (Appendix A) while still at the field site. Where necessary, on action notes were added to the sheet laterthese were annotated so as to clearly distinguish them from the in action notes. Amy completed each sheet by hand then typed up her notes within 24 hours. At the end of the period, once data had been collected, coded and analysed, a final meta-reflective account was written. This involved Amy recording her thoughts on the application of reflective practice to her fieldwork. In doing this, Amy attempted to 'step back' and reflect upon the findings and what potential she could see for the application of reflective practice to field biology. Thoughts were recorded by hand, as they occurred, and subsequently typed up. 


\section{Data analysis}

Initial data analysis began once all eight data sheets were completed, and consisted of an overview of all recorded reflections. For each paired visit, the words recorded under each domain (cognitive, psychomotor, affective and conative) were counted and coded. This allowed the identification of any obvious differences in amount and type of data between domains and visits.

Since the data produced was qualitative, we used content analysis to find key messages within the data. A mixed approach using emergent and template coding was developed. Using emergent coding we coded the data collection sheets using the open coding and axial coding phases of Grounded Theory Methodology (Strauss \& Corbin, 1998). During the open coding phase, sections of text were labelled according to themes that emerged from scrutiny of the logged reflections. During this phase, we both coded the data separately to reduce intra-coder bias. After this was complete we met and juxtaposed our results. At this stage we used axial coding, where our individual themes were compared, categorised and 'related to their subcategories to form more precise and complete explanations' (Strauss \& Corbin, 1998, p.24). From this we were able to develop four coding templates: one for each of the domains of the VR model. The cognitive template was made up of 11 codes; the conative template had 12 codes; the affective template had 11 codes, and the psychomotor template had six codes. These templates were then applied to the data afresh by both of us. Inter-coder reliability was high; any differences were small and easily resolved after a short discussion. This inter-coder iterative approach meant that the tool that gave voice to the data was developed from the data itself rather than simply imposed upon it.

\section{Ethics and limitations}

Potential ethical concerns associated with the data collection process were considered. These included impact on colleagues - potential subjects of the reflective comments, and on the quality of fieldwork data - for example as a result of the reflection acting as a distraction from the fieldwork itself. However, both of these were deemed to be minimal. Prior to data collection, the task was briefly described to curious fieldwork colleagues, who were happy to continue as normal and focus on the job in hand. The notes 
tended to be made during natural breaks in the fieldwork tasks, so did not interfere with the routine of biological data collection. The reflection process did not have any noticeable effect on the time taken to complete each site. Limitations of this study include the fact that it examines just one example of a fieldwork project, and the experiences of one particular field practitioner. Every fieldwork-based project will be associated with a different set of challenges and events to reflect upon. Similarly, different field researchers will respond to these challenges and events in different ways. Even given the fairly structured, balanced approach of VR, people may still vary in the way that they embrace reflective practice. However, we feel that there are also many universal lessons that can be gleaned from a study such as ours, and that the nature of the challenges and conditions faced in this fieldwork will be common to many other projects, even if the specifics are not.

\section{Data and data analysis}

During content analysis, the four domains were examined individually. For each, the results of the application of each coding template are presented in table form, and the most frequently coded themes are reported and supported with examples. Emerging patterns and interesting associations within and between the most frequently coded themes are discussed within each domain. Here we present key findings under each of the four domain headings: cognitive, conative, affective and psychomotor. Each section highlights individual themes that were found to be significant within a particular domain. The significance of the most heavily coded themes is discussed with reference to specific reflective comments. This section closes with a meta-reflective account - where Amy reflects on her experience of using the VR model during fieldwork. In all, we present what was found to be significant; examples of significant areas, and analysis of what such data might mean.

\section{The cognitive domain}

Cognitive was the most heavily coded of the four domains. The theme that arose most frequently was 'Assessing environmental characteristics', with nine instances (see Table 1). This template code was 
dominated by observations relating to water level, for example: 'Water level quite high' and 'Water level is lower than usual', as well as other features of the environment that could vary from visit to visit, such as 'Highly 'disturbed' site with black anoxic substrate'. These reflections emphasise the need for the field researcher to assess the physical conditions of a site on arrival, in order that appropriate adjustments to the protocol might be made. These observations also highlight the importance of prior experience, as assessments such as 'quite high' and 'lower than usual' are relative, and rely on an understanding of what is 'normal' for that particular habitat. After this, 'Assessing biological characteristics' and 'Adjustments to sampling protocol' were the second most commonly identified codes. The former consisted of statements relating to the fauna being surveyed, and included both unusual observations of individual specimens, such as 'Caught one strange fish, which couldn't be easily identified-maybe a feral or hybrid form', as well as thoughts on the more general patterns of biodiversity. Observations like this are data in themselves, and represent the qualitative information that can be lost during standard quantitative data collection. Although somewhat a distraction from the research questions being addressed, these are potentially valuable observations for future research or data interpretation. 'Adjustments to sampling protocol' applied to statements where observations led to changes in procedure. For example the reflection 'Had to make a decision about measurement of one of the transects due to the river changing course a little' is in response to an environmental characteristic, and 'Have to adjust "flow" methods here to account for a section of rapids' related to the physical nature of the river. Cognitive reflections such as these often occur subconsciously in the experienced practitioner, and are a normal part of fieldwork. However, there may be value in explicitly registering them and acknowledging the flexibility that has to be incorporated into fieldwork protocols. Inexperienced field researchers, trained in rigour and precision as scientists, may be panicked the first time that fieldwork protocol has to be adjusted in situ. Therefore, less-experienced field researchers may find the introduction of reflective practice reassuring, as it would encourage such adjustments to be made explicit and in doing so emphasise that they are integral to successful fieldwork. 
Together, the most common codes here reflect that Amy's most important cognitive concerns during fieldwork tend to be related to the flexibility that is required in response to different conditions. The reason for doing fieldwork as opposed to controlled laboratory experiments in biology is to capture a more realistic view of what happens in natural ecosystems, but with this comes a large amount of natural variation too, both in the data being collected and in the environment the fieldworker is faced with. To arrive at a site and identify the environmental and biological characteristics that may require adjustments, and then to make these changes, is a vital cognitive process in successful data collection.

Table 1. Application of cognitive coding template

\begin{tabular}{|l|r|}
\hline Code & $\begin{array}{r}\text { No. of } \\
\text { codings }\end{array}$ \\
\hline Assessing environmental characteristics & 9 \\
\hline Assessing biological characteristics & 7 \\
\hline Adjustments to sampling protocol & 7 \\
\hline Problematic environment for study & 5 \\
\hline Conditions lead to reflection on study design & 5 \\
\hline Professional decisions & 5 \\
\hline Ideal environment for study & 4 \\
\hline Field-based decision & 4 \\
\hline Difficulty of multi-tasking & 2 \\
\hline Managing team activity & 2 \\
\hline Safety concerns & 1 \\
\hline & 51 \\
\hline
\end{tabular}

\section{The conative domain}

In total, 43 aspects of data were coded as relating to the conative domain (see Table 2). Within this, the highest scored category related to the 'Positive attitude' of the researcher. Comments such as 'Everyone in good spirits and seems to have right attitude' and 'feeling more energetic' show a link between the fieldwork environment and Amy's natural tendencies. Almost one fifth of the reflections on conation relate to Amy 'feeling motivated' in the field. In this instance, reflection on the conative domain has highlighted how biological fieldwork is more than just a mechanical activity and that there is a relationship between attitude and effort in the field. This is perhaps something we intuitively know but through recording such reflections individuals can get a sense of this relationship at the personal level. 
Three other template codes showed relatively high scores - 'Impact of absentmindedness'; 'Conditions affect approach to work', and 'Happy to be in the field'. The first code here suggests that, in order to do a good job in the field, the practice of the researcher needs to be examined to limit human error and we can see this in the reflection: 'Have to keep thinking and double checking where we've got to and what needs doing'. Further, Amy shows that in order to deal with possible absentmindedness, she is willing to put in extra effort: 'I forgot to take photos so had to run back at the last minute'. The connection between field conditions and the amount of effort applied is shown in the five codings for 'Conditions affect approach to work'. These codings show that whilst conditions can stop fieldwork: 'Had to stop for a rain shower - cold and wet!' At other times, the reflect accounts show that Amy is willing to put in extra effort to get the job done. There does seem to be some link between the directed effort of the researcher and the feelings that are drawn from being in the field. Five aspects of data were coded as showing Amy was 'Happy to be in the field' and the reflections that she had a 'nice feeling' about being on site and that she was 'Happy to be up and out in the field' suggest a link between feeling and fieldwork.

Table 2. Application of conative coding template

\begin{tabular}{|l|r|}
\hline Codes & $\begin{array}{r}\text { No. of } \\
\text { codings }\end{array}$ \\
\hline Positive attitude & 8 \\
\hline Impact of absentmindedness & 5 \\
\hline Conditions affect approach to work & 5 \\
\hline Happy to be in the field & 5 \\
\hline Overcoming a lack of enthusiasm and/or energy & 4 \\
\hline Distraction & 4 \\
\hline Deviating from 'auto-pilot' & 4 \\
\hline Supporting colleagues & 2 \\
\hline Wanting to do a good job & 2 \\
\hline Delegation & 2 \\
\hline Frustration & 1 \\
\hline Working on 'auto-pilot' & 1 \\
\hline & total \\
\hline
\end{tabular}




\section{The affective domain}

Reflections relating to the affective domain showed the lowest number of codings - only 30 aspects of data were coded in total (see Table 3). The inter- and intra-rater reviews built into the methodology suggest that this is unlikely to be an error in the coding process, but this low number suggests that something is occurring here. Another factor worth noting is that the actual passages of data that were coded tended to be longer than those in the other domains. Therefore, it could be suggested that attitudes and emotions were evident in the reflections but that they take more words to describe.

The two template codes with the highest number of codings - 'Negative distractions overcome' and 'Affected by human activity at site' show how feelings regarding extraneous matters can impinge upon fieldwork but that they can be set aside so that the research can continue. The key evidence of overcoming negative distractions came from reflections such as 'Fumes from the generator bother me today, and made me change my location for some tasks' and 'Have no choice but to be in the sun for most tasks'. Evidence of human activity stirred affected comments such as, 'On arrival we disturbed a religious ritual, probably a Spiritual Baptist ceremony. Discovered a dead charred goat on a recent fire pile-apparently part of a sacrifice' and 'People came to extract water while we surveyed - not sure if allowed but had to ignore and stay focussed'. In all these instances negative emotions are overcome, either by making physical adjustments, such as changing location, or by psychologically shifting focus away from the distraction. However, not all codings on the affective domain related to negative feelings that had to be kept in check - some were emotionally balanced, and seven related to positive feelings. The code 'Field aesthetics increase happiness' is evidence of this with comments such as 'I am happy to be working here', and activity on one survey site being described as 'enjoyable'.

It would seem that, despite the low number of codings and a slight bias towards reflections that code for negative emotions, a range of attitudes are at play during fieldwork and that these can be kept in balance, where necessary, through professional conduct. 
Table 3. Application of affective coding template

\begin{tabular}{|l|r|}
\hline Codes & $\begin{array}{c}\text { No. of } \\
\text { codings }\end{array}$ \\
\hline Negative distractions overcome & 5 \\
\hline Affected by human activity at site & 5 \\
\hline Field aesthetics increase happiness & 4 \\
\hline Problematic conditions & 3 \\
\hline Temporal reflection & 3 \\
\hline Positive distractions overcome & 2 \\
\hline Interaction with local people & 2 \\
\hline Professional adjustment & 2 \\
\hline Ethical reflection & 2 \\
\hline Unsettled by previous activity & 1 \\
\hline Takes time over beautiful site & 1 \\
\hline & total \\
\hline
\end{tabular}

\section{The psychomotor domain}

A total of 49 passages were identified with respect to the psychomotor template codes (see Table 4). It is interesting that this domain is at least as well represented in terms of scoring as the others, despite notably fewer template codes. This suggests that although psychomotor concerns may be less varied, they are no less important to fieldwork and reflective practice in fieldwork.

By far the most frequently coded template code was 'Physical environment presents challenge', with more than $30 \%$ of the total scorings. Examples include both instances where physical structures presented a challenge: 'Have to lean over bank with oxygen and $\mathrm{pH}$ meters' and where more general conditions and terrain make the work more difficult: 'Hard to move from one end to the other'. These reflections show the reality of fieldwork through highlighting the nexus between the physicality of biological fieldwork and the nature of the environment itself. Through reflections such as these we can see the particular mindset that is required to complete academic work in the natural environment and we can begin to see how this might differ from such activity in a controlled laboratory environment.

The second highest scoring template code was 'Change in body position or posture'. Most passages here were in response to the physical environment necessitating adjustments to body form or posture. For example 'Taking the photos here involves scrambling down the bank and passing (ducking) 
under a bridge' and 'plenty of ducking, bending and climbing involved'. This was closely followed by 'Safety concerns' - which tended to relate to the conditions of the terrain and taking extra care in response to this, such as 'Very slippery ground - I slipped once, but was not hurt' and 'Slippery in parts as have to navigate rocks -I am cautious and careful as a result'. These reflections are important, as such details will likely be left out of the final reports that will be produced on the project. While the emphasis of the fieldwork itself is to examine the tropical mountain stream of Trinidad, two important points may be learned and generalised. Firstly, if researchers are to build upon this study and run follow-up fieldwork studies, then they may benefit from insider knowledge that enhances their experiences within such an environment. Secondly, these reflections will support Amy's future development as she is able to record and reflect upon her experiences as well as her data.

Fieldwork, especially biological fieldwork, is by its nature a physical activity, and often requires working under challenging physical conditions. It is therefore unsurprising that the psychomotor domain is well-represented in its reflective practice. Critical to successful fieldwork is finding ways to collect data despite these challenges and, as such, reflections within this domain could prove particularly fruitful when applied to fieldwork.

Table 4. Application of psychomotor coding template

\begin{tabular}{|l|r|}
\hline Codes & $\begin{array}{c}\text { No. of } \\
\text { codings }\end{array}$ \\
\hline Physical environment presents challenge & 16 \\
\hline Change in body position or posture & 10 \\
\hline Safety concerns & 9 \\
\hline Physical environment makes things easier & 7 \\
\hline Conditions make equipment handling difficult & 4 \\
\hline Feeling uncomfortable & 3 \\
\hline \multicolumn{2}{|c|}{ total } \\
\hline
\end{tabular}




\section{Meta-reflective account}

At the end of the research period, Amy completed a final meta-reflective account regarding her thoughts on the application of the VR model to the fieldwork:

Fieldwork can be an exhausting experience, and for me, the results of reflective practice have helped me to realise why this is: despite fieldwork appearing to be largely physical with the odd cognitive consideration, in reality your brain is working on many different things concurrently, as demonstrated by the spread of data throughout all four domains - affective, conative, physical and cognitive. Previous to this study, I don't think I was conscious of the multiple ways in which fieldwork was working my brain.

My experience during this study has convinced me that there would be potential benefits to including a more formal reflective process at the start of future field studies. Indeed, the main change I would make would be to employ reflection earlier on in the project. This is because I believe the greatest benefits would come from reflecting at the planning stage, where there is still room for real adjustments that may emerge as necessary during the reflective process, and so that these can implemented without conflicting with the scientific rigour and repeatability of the methods and design. One of the most important things when beginning a new study - especially a long term study - is to get everything right at the start, so that minimal adjustments to methods are required during the study and data are completely comparable over time. In this way, reflection has the potential to actually improve the quality of data being collected. Additionally, certain aspects of fieldwork such as the delegation of tasks can be implemented at any stage of a study with minimal implications for consistency of data collection, and reflection is one way to identify areas where delegation may be useful.

It would be interesting to survey scientists for their attitudes towards reflective practice, as I suspect many, like me before this study, would not even have a concept of what it involves, and that even after an explanation they would be dismissive/sceptical of its application to 
scientific fieldwork. Overall, reflective practice has provided me with insight into the scientific process - one's ability able to stay balanced and not be affected by feelings and moods associated with being out in the field at a particular site on a particular day.

\section{Discussion}

In developing this study a number of factors were considered: the model used to record the fieldwork reflections needed to be portable; reflections needed to be recorded against a number of domains, and the model needed to be flexible and practical for use in the field. The Vitruvian Reflection framework was able to address these factors and our analysis of its application to biological fieldwork has brought a number of specific insights. Our data would suggest there is clear evidence for the further exploration and development of reflective practice within biological fieldwork. Reflection may already be occurring within this practice but there was no sign of this in the literature. By applying an appropriate reflective framework we were able to access valuable practitioner data that may never have been made explicit otherwise (Loughram, 2002; Drummer et al, 2008). We can see an example of this in Amy's metareflective comment that 'I don't think I was conscious of the multiple ways in which fieldwork was working my brain'. Here the suggestion is not that she was not processing all this information before, rather that she was not always aware of this perceptive activity. By applying the VR framework we were able to make explicit that which had previously been implicit, thus consciously framing the relationship between the practitioner and their environment - something that Loughram (2002) considers to be essential to effective reflective practice.

Scott et al (2011) claim that biological fieldwork covers many domains of learning. This study was able to support this claim and demonstrate how a structured reflective approach can highlight the interconnectedness of the domains of learning in relation to biological fieldwork and can offer a nuanced account of fieldwork. Since the overall outcome of the study shows a broad range of reflective comments, there does seem to be evidence that reflective practice and biological fieldwork are not the uneasy bedfellows that the (lack of) literature might imply. Drummer et al (2008) discuss the link between 
reflection and development within a specific context. If, as this work suggests, reflection is appropriate to the context of biological fieldwork, then it can be suggested that this might lead to the further development of practice within this area.

The application of VR was able to support Fazey et al's (2006) position on the need for flexibility, judgement and adaptation in fieldwork in order to respond to the various conditions experienced. Through logged reflective accounts we were able to access evidence of particular intellectual activities that were at play during the fieldwork, and, by examining such activity, practitioners in the field might be better informed about why they make certain judgements. Evidence of this came from all four domains examined and demonstrates the relationship between the physical and the intellectual aspects of fieldwork. The VR framework was also able to illustrate the role of motivation and the link between the feelings and the actions of the researcher in the field. Such findings may have been found through reflection on Critical Incidents (Griffin, 2003) or through the review of video footage (Deaton, 2012); however, these approaches lack the flexibility that is needed in the field. Therefore the VR model was found to be an effective tool for the situation under study. Clinton (1997) comments that there will always be some conflict in the analysis of written reflections, but such a point is true of any reflective model that involves writing and is, therefore, limited in its significance. Overall, we found that the VR model was able to support balanced reflection during biological fieldwork and was able to suggest how colleagues working in the natural sciences can begin to embrace reflective practice. A powerful instrument may be overlooked if researchers in the field are not given the opportunity, tools and legitimacy to 'critically problematise' their practice (Schön, 1992).

\section{Conclusion}

As far as we are aware, our study represents the first time that a balanced reflective practice framework has been applied to fieldwork in the life sciences. The fieldwork in this instance was biodiversity surveys in tropical mountain streams of Trinidad in the Caribbean, but most projects face similar challenges. This case study is fairly typical of biological fieldwork in that it requires adjustment of actions and methods 
depending on the particular physical and social conditions, both of which can change with each trip. In some ways, the essential 'repeatability' of scientific experiments limits the scope for ongoing improvements as a result of critical reflections. However, this study has shown that even within the constraints of a long-term study where the methods are already well defined, there is still room for reflection.

Overall, there seems to be little reason for the absence of reflective practice in biological fieldwork. However, it should be clarified that just because reflection is not formally practiced in this field, does not mean that scientists are not employing any form of reflection at all. The question is whether biological field researchers are missing a developmental trick if they are not exposed to the possibilities of reflective practice, and whether there is a place for reflective practice to be promoted and formalised as a useful tool in biological fieldwork, just as it is central to many fields in the social sciences. Instead of considering the environment and the researcher's mind as two separate entities there is room to consider how the environment is experienced by the researcher (Fazey \& Marton, 2002) and our findings suggest that the introduction of a model of balanced reflective practice to biological fieldwork could support this holistic approach.

\section{References}

Blair, E. (2011). Balanced reflection as a means of practitioner development in the Post-Compulsory Education and Training Sector. Research in Post-compulsory Education: Special Issue on 'Transformation in Post-Compulsory Education, 16 (2), 249-261.

Bloom, B., Englehart, M. Furst, E., Hill, W. \& Krathwohl, D. (1956). Taxonomy of educational objectives: The classification of educational goals. Handbook I: Cognitive domain. New York, Toronto: Longmans, Green.

Clinton, M. (1998). On reflection in action: Unaddressed issues in refocussing the debate on reflective practice. International Journal of Nursing Practice, 4, 197-202.

Darwin, C.R. (1839). Narrative of the surveying voyages of His Majesty's Ships Adventure and Beagle between the years 1826 and 1836, describing their examination of the southern shores of South America, and the Beagle's circumnavigation of the globe. Journal and remarks. 1832-1836. London: Henry Colburn. 
Deaton, C. (2012). Examining the use of a reflection framework to guide teachers' video analysis of their science teaching practice. Electronic Journal of Science Education, 16(2). Retrieved from http://ejse.southwestern.edu/article/view/9054/7970

Drummer, T.J.B., Cook, I.G., Parker, S.L., Barrett, G.A. \& Hull, A.P. (2008) Promoting and assessing 'Deep Learning' in geography fieldwork: An evaluation of reflective field diaries. Journal of Geography in Higher Education, 32(3), 459-479.

Dyment, J.E. \& O'Connell, T.S. (2010). The quality of reflection in student journals: A review of limiting and enabling factors, Innovations in Higher Education, 35, 233-244.

Eisner, E. \& Powell, K. (2002). Art in science? Curriculum Inquiry, 32(20), 131-159.

Fazey, I., Fazey, J.A \& Fazey, D.M.A. (2005 ). Learning more effectively from experience. Ecology and Society, 10(2), 4. Retrieved from http://www.ecologyandsociety.org/vol10/iss2/art4/

Fazey, I., Proust, K., Newell, B., Johnson, B. \& Fazey, J.A. (2006). Eliciting the implicit knowledge and perceptions of on-ground conservation managers of the Macquarie Marshes. Ecology and Society, 11(1), 25. Retrieved from: http://www.ecologyandsociety.org/vol11/iss1/art25/

Fazey, J.A. \& Marton, F. (2002). Understanding the space of experiential variation. Active Learning in Higher Education, 3(3), 234-250.

Griffin, M.L. (2003). Using Critical Incidents to promote and assess reflective thinking in preservice teachers. Reflective Practice, 4(2), 207-220.

Hays, R. \& Gay, S. (2011). Reflection or 'pre-reflection': What are we actually measuring in reflective practice? Medical Education, 45, 116-118.

Hazzan, O. \& Tomayko, J. (2004). Reflection processes in the teaching and learning of human aspects of software engineering, in proceedings of the 17th Conference on Software Engineering Education and Training, 1-3 March 2004, Norfolk, VA, USA.

Higgitt, M. (1996). Addressing the new agenda for fieldwork in higher education. Journal of Geography in Higher Education, 20, 391-398.

Kent, M. Gilbertson, D. \& Hunt, C. (1997). Fieldwork in geography teaching: A critical review of the literature and approaches. Journal of Geography in Higher Education, 21, 313-332.

Krathwohl, D., Bloom, B.S. \& Masia, B. B. (1964) Taxonomy of Educational Objectives, the classification of educational goals-Handbook II: Affective Domain. New York: McKay

Levine, R.B., Kern, D.E. \& Wright, S.M. (2008). The impact of prompted narrative writing during internship on reflective practice: a qualitative study. Advances in Health Science Education, 13, 723-733.

Lock, R. (1998). Fieldwork in the life sciences. International Journal of Science Education, 20(6), 633642.

Loughran, J.J. (2002). Effective reflective practice: In search of meaning in learning about teaching. Journal of Teacher Education, 53, 33-44. 
Mair, C. (2012). Using technology for enhancing reflective writing, metacognition and learning. Journal of Further and Higher Education, 36, 147-167.

Mann, K., Gordon, J. \& MacLeod, A. (2009). Reflection and reflective practice in health professions education: a systematic review. Advances in Health Sciences Education, 14(4), 595-621.

McCollum, S. (2002). The reflective framework for teaching physical education. Journal of Physical Education, Recreation \& Dance, 73(6), 39-42.

McGuinness, M. \& Simms, D. (2005) Going global: long-haul fieldwork on undergraduate geography. Journal of Geography in Higher Education, 29(2), 241-253.

Noss, R. F. (1996). The naturalists are dying off. Conservation biology, 10(1), 1-3.

Schön, D.A. (1983). The reflective practitioner. New York: Basic Books

Schön, D.A. (1992). The theory of inquiry: Dewey's legacy to education. Curriculum Inquiry, 22(2), 119139.

Scott, G. W., Goulder, R., Wheeler, P., Scott, L. J., Tobin, M. L., \& Marsham, S. (2012). The value of fieldwork in life and environmental sciences in the context of higher education: a case study in learning about biodiversity. Journal of Science Education and Technology, 21(1), 11-21.

Smith, E. (2011). Teaching critical reflection. Teaching in Higher Education, 16, 211-223.

Strauss, A. \& Corbin, J. (1998). Basics of qualitative research: Techniques and procedures for developing grounded theory (2nd ed.). Thousand Oaks, CA: Sage.

Thompson, N. \& Pascal, J. (2012). Developing critically reflective practice. Reflective Practice, 13(2), 311-325.

Thorpe, K. (2004) Reflective learning journals: From concept to practice. Reflective Practice, 5(3), 327343.

Thorsen, C.A. \& DeVore, S. (2013). Analyzing reflection on/for action: A new approach. Reflective Practice, 14(1), 88-103.

Wallace, A.R. (1889). A narrative of travels on the Amazon and Rio Negro: with an account of the native tribes, and observations on the climate, geology, and natural history of the Amazon Valley (No. 8). Ward, Lock and Company. 
Appendix A. Example of data collection sheet

Psychomotor
How do you proceed physically within this
site?
(Where do you stand within the site? How do
you manipulate instruments? What is your
body language saying?)

Conative

How much effort will you give to this site?

(How do you feel generally today? Are you making a special effort? Are you working on auto-pilot? How badly do you want results?)

\section{Cognitive}

What are your 'expert' thoughts about this site?

(Is it going to yield good results? What is your training/experience telling you? What are the issues?)

\section{Affective}

How do you feel about this site?

(Does this site have any aesthetic qualities? Can you separate your humanity from your scientific objectivity 
Appendix B. Example showing six versions of data collection sheet

(1)

\begin{tabular}{|l|l|}
\hline Cognitive & Conative \\
& \\
\hline Affective & Psychomotor \\
& \\
\hline
\end{tabular}

(3)

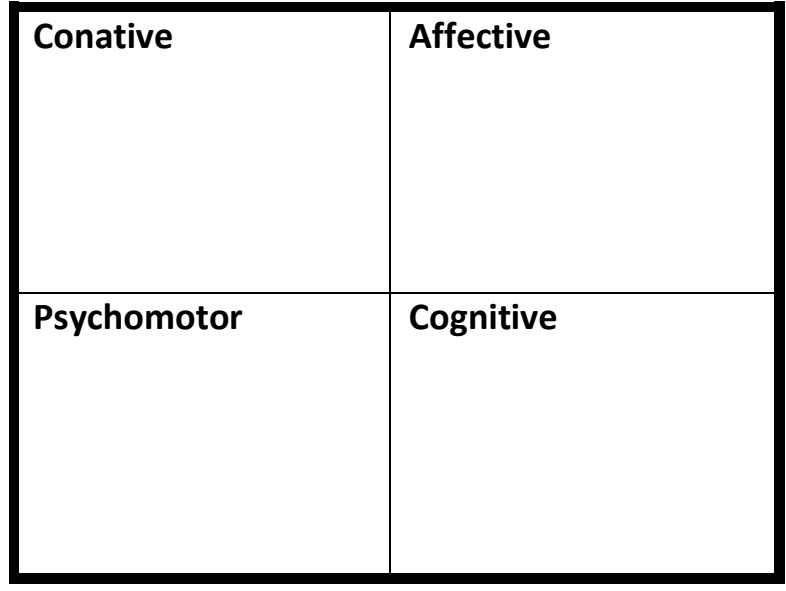

(5)

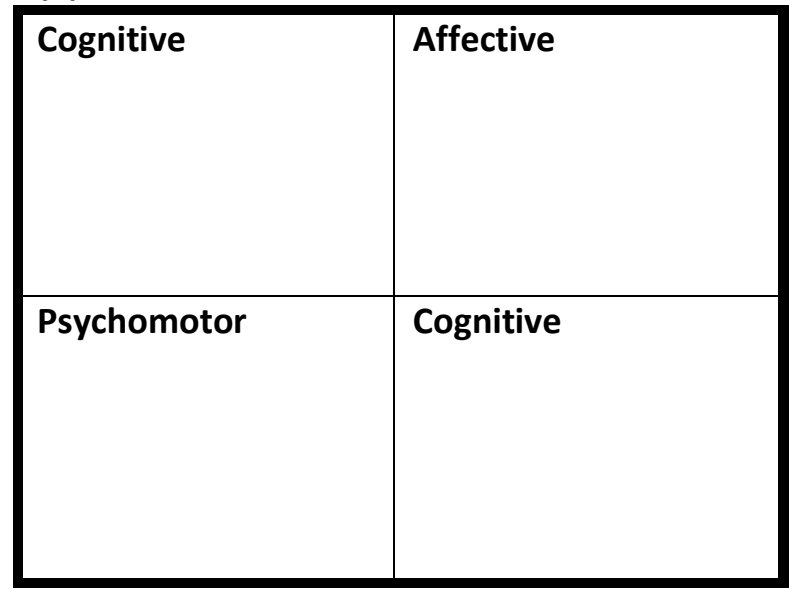

(2)

\begin{tabular}{|l|l|}
\hline Psychomotor & Affective \\
\hline Conative & Cognitive \\
& \\
\hline
\end{tabular}

(4)

\begin{tabular}{|l|l|}
\hline Affective & Psychomotor \\
& \\
\hline Cognitive & Conative \\
& \\
\hline
\end{tabular}

(6)

\begin{tabular}{|l|l|}
\hline Psychomotor & Conative \\
& \\
\hline Affective & Conative \\
& \\
& \\
\hline
\end{tabular}

